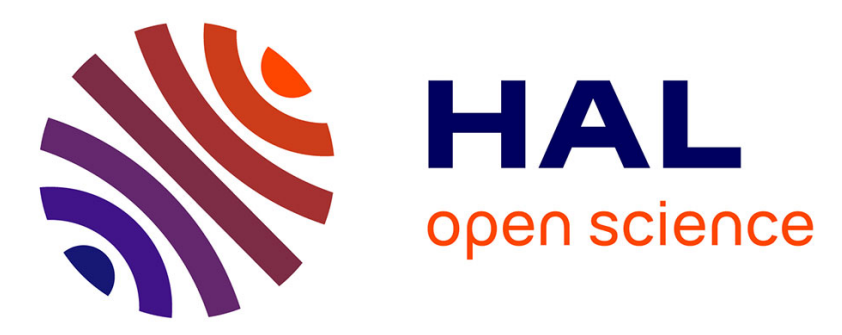

\title{
La science économique comme idéologie. La science de gestion comme viatique de l'actionnaire
}

\author{
Eric Brunat, Jacques Fontanel
}

\section{To cite this version:}

Eric Brunat, Jacques Fontanel. La science économique comme idéologie. La science de gestion comme viatique de l'actionnaire. Marché et Organisations, 2021, Crise pandémique, crise globale: réalité et controverses théoriques, 41, pp.59-77. hal-03232720

\section{HAL Id: hal-03232720 \\ https://hal.univ-grenoble-alpes.fr/hal-03232720}

Submitted on 22 May 2021

HAL is a multi-disciplinary open access archive for the deposit and dissemination of scientific research documents, whether they are published or not. The documents may come from teaching and research institutions in France or abroad, or from public or private research centers.
L'archive ouverte pluridisciplinaire HAL, est destinée au dépôt et à la diffusion de documents scientifiques de niveau recherche, publiés ou non, émanant des établissements d'enseignement et de recherche français ou étrangers, des laboratoires publics ou privés. 


\title{
La science économique comme idéologie La science de gestion comme viatique de l'actionnaire
}

\author{
Éric Brunat et Jacques Fontanel \\ Revue Marché et Organisations $n^{\circ} 41$ \\ 2021
}

Depuis la généralisation de l'économie de marché, la science économique a sonné le glas de l'économie politique. Dans ce contexte, le marché était omnipotent et l'Etat devait concentrer son activité sur l'organisation d'un domaine régalien, souvent fluctuant et plutôt régressif en fonction des intérêts privés. La science du management elle-même a donné des outils aux entreprises dans le cadre d'un système économique qui prônait l'intérêt individuel comme objectif déterminant pour chacun des acteurs économiques. Aujourd'hui, avec la pandémie de Covid-19, les analyses des économistes libéraux deviennent obsolètes face à une profonde crise économique et sociale. L'économie révèle désormais son caractère éminemment politique. Les secteurs publics internationaux, nationaux et locaux organisent la lutte contre la crise de l'économie de marché. L'État redevient l'acteur central de la gestion de l'économie nationale, et il est alors amené à faire des choix politiques qui redessinent les structures économiques et sociales du pays, notamment face aux grandes firmes multinationales et aux ambitions des autres nations. Le caractère profondément politique et social d'une économie mondialisée est mis en évidence par cette crise, qui se caractérise par la violence des relations entre les États et entre les citoyens.

Since the generalisation of the market economy, economic science has sounded the death knell for political economy. In this context, the market was omnipotent and the State had to concentrate its activity on the organisation of a regalian domain, often fluctuating and rather regressing according to private interests. Management science itself gave tools to companies within the framework of an economic system that advocated individual interest as the determining objective for each of the economic actors. Today, with the Covid-19 pandemic, the analyses of liberal economists are becoming obsolete in the face of a deep economic and social crisis. The economy is now revealing its eminently political character. The international, national and local public sectors organised the fight against the crisis of the market economy. The state once again became the central actor in the management of the national economy, and it was then led to make political choices that would redraw the economic and social structures of the country, particularly in the face of the large multinational firms and the ambitions of other nations. The profoundly political and social character of a globalised economy is highlighted by this crisis, which is characterised by the violence of relations between states and between citizens.

science économique, science de gestion, politique économique, firmes multinationales, capitalisme financier, pandémie, Covid-19, inégalité sociale

economics, management science, economic policy, multinational firms, financial capitalism, pandemic, Covid-19, social inequality 


\section{Introduction}

Une société est fondée sur un ensemble de dispositifs juridiques et d'intérêts communs, souvent organisé autour d'une Constitution. Elle définit le fonctionnement d'une vie commune et le mode d'organisation qui assoit l'exercice du pouvoir ou des pouvoirs. Sous l'effet de la Covid-19, la globalisation, qui avait déjà tendance à stagner en pourcentage du PIB mondial, va connaître, au moins conjoncturellement, une récession. Pour lutter contre la pandémie, les règles fixées par l'État s'imposent à l'intérieur du périmètre de son territoire, même si, dans les institutions internationales d'intégration, des règles peuvent être mutuellement définies. Aujourd'hui, un intérêt particulier semble être porté à une politique économique publique plus respectueuse des questions environnementales, soucieuse des contraintes liées à l'épuisement des ressources naturelles et clairement concernée par une reterritorialisation des activités économiques et sociétales essentielles à la Nation, pour définir un développement socialement et économiquement durable, dans le respect de normes environnementales soutenables. Les théories de l'économie institutionnelle reviennent sur le devant de la scène, comme à chaque fois qu'une crise économique impacte tous les acteurs nationaux et internationaux.

Pour l'analyse néolibérale, l'intérêt personnel constitue la base du bon fonctionnement de l'économie de marché, car il conduit à une situation jugée, in fine, optimale et innovatrice. Pour Adam Smith, la «main invisible», fondée sur l'intérêt individuel, constitue la clef du développement économique. De ce fait, pour des raisons économiques principalement, il condamne l'esclavage ${ }^{1}$, la colonisation, les monopoles et cartels, les droits de douane et il prônait le respect des lois édictées par un État dépositaire des fonctions régaliennes. Les néoclassiques ont repris implicitement ce terme dans le modèle de la concurrence pure et parfaite, en insistant sur la rationalité des décisions individuelles. Depuis, il a été observé empiriquement et démontré par la théorie des jeux que cette intuition n'était pas justifiée, que dans tous les cas, des situations de concertation et de coopération étaient plus efficaces à court terme et à long terme. Pourtant, inconsciemment, les économistes néoclassiques continuent à se référer de fait à la "main invisible ", expression qui a été reprise officiellement par le Président Bush lui-même pendant son mandat.

En parodiant Churchill, on pourrait dire que le capitalisme est le pire des systèmes, à l'exception de tous les autres, ce qui n'est pas vraiment une démonstration scientifique. La protection de l'intérêt individuel est souvent confirmée dans les Constitutions. Mais si, comme le pensait Sartre, "l'enfer c'est les autres », il est donc nécessaire de protéger les citoyens contre les abus de tous ordres. Par exemple, le respect de la liberté d'entreprendre est un choix constitutionnel qui peut à tout moment être remis en cause dans le cadre d'un changement de société. En effet, il n'y a pas de droits préalables, sans définition des obligations individuelles et collectives de chaque agent économique à l'égard de la communauté qui garantit ses droits. Lorsque les $\mathrm{GAFAM}^{2}$ et autres firmes multinationales utilisent les infrastructures nationales ou internationales en optimisant les divergences des cadres juridiques, fiscaux, sociaux et des conditions de travail du monde entier sans se sentir responsables socialement des avantages qui leur sont ainsi procurés, elles sont concentrées seulement sur la maximisation de leurs bénéfices immédiats. Elles se comportent alors comme des passagers clandestins ou comme des passagers logés en première classe mais qui ne paient

\footnotetext{
${ }^{1}$ «L'ouvrage fait par des mains libres revient définitivement à meilleur compte que celui qui est fait par des esclaves » A. Smith (1776), La richesse des nations, Chapitre 8, Guillaumin, réédition de 1843 (première édition en 1776), tome I, p. 84-119. A noter que cette théorie a été confirmée par Robert Fogel (prix Nobel d'économie 1993) qui a démontré que, nonobstant la question de la liberté, au XIXe siècle, les esclaves du Sud étaient mieux traités et avaient un niveau de vie supérieur à celui des ouvriers du Nord des USA.

${ }^{2}$ Google, Amazon, Facebook, Apple, Microsoft
} 
que les tarifs les plus bas possibles. Le principe du « laissez-faire » suppose que l'État agisse comme un gendarme appelé à faire respecter la propriété privée, le droit d'entreprendre et la liberté d'agir, sans se poser la question de ce que doit l'individu au social. De fait, lorsque les entreprises contestent les impôts sur les sociétés, la raison n'est pas seulement fondée sur la force de leur position concurrentielle mondiale, elle est aussi une expression parfaite de l'intérêt individuel des dirigeants et actionnaires qui souhaitent engranger le maximum de profits et de revenus après les prélèvements publics obligatoires. Pourtant, peu d'études ont été développées par la recherche économique sur ce thème, faute, entre autres?, d'informations statistiques et fiscales précises. Aujourd'hui, de nouveaux chercheurs s'engagent dans cette voie et mettent en évidence l'importance des questions fiscales dans les ressorts des inégalités sociales dans le monde (Saez et Zucman, 2020 ; Piketty, 2019).

Dans une économie de marché globalisée, le rôle de l'Etat est limité par ses adhésions aux organisations économiques internationales et il est contesté par les firmes multinationales ce qui réduit son action régalienne. L'apport des économistes pour définir les rôles respectifs des acteurs économiques fait dès lors l'objet de controverses, dans le temps et dans l'espace. La diversité des théories économiques témoigne des difficultés réelles d'émergence d'une science économique unitaire, tant les hypothèses et postulats de départ retenus sont malheureusement souvent éloignés de la réalité des faits.

\section{1) Le rôle contesté de l'État dans l'économie de marché}

Dans une économie de marché, l'État doit aider les entreprises à prospérer et intervenir lorsque la crise économique s'invite. Avec l'essor de la globalisation, les entreprises ont délocalisé leurs activités, elles ont engagé des processus de management à flux tendus et elles ont exercé leurs libertés dans le cadre des lois spécifiques des pays où elles exercent. Les États sont souvent corsetés par des règles fixées par les organisations internationales idoines dont ils sont membres. Dans ces conditions, les responsables publics sont jugés non seulement pour les actions dont ils ont la responsabilité directe, mais aussi sur la base d'opérations économiques qui, dans un système d'économie de marché, échappent largement à leur pouvoir. Les instruments dont ils disposent pour infléchir les résultats économiques nationaux sont de plus en plus réduits, contestables, contestés, conduisant à des comportements d'aléa moral des entreprises difficiles à gérer, notamment dans le cadre fiscal. La globalisation remplit son œuvre, celui de réduire les interventions économiques des autorités publiques sur leurs territoires.

Avec la pandémie du coronavirus, l'État devient un chef de guerre, celui qui assure la survie des citoyens, celui qui est capable d'interrompre l'économie pour combattre la mort. Cependant, force est de reconnaître qu'il sera vite fragilisé par le poids de la dette et la violence de la crise économique. La souveraineté des États redevient ainsi un thème majeur de la vie géopolitique d'aujourd'hui. Les unions régionales fragilisent parfois les gouvernements d'États désireux d'agir dans un contexte économique perturbé, mais limités par les engagements internationaux préalables. A titre d'illustration, dans la lutte contre la Covid-19, l'action de l'Union européenne est souvent freinée par l'opposition entre les pays du Nord et les pays du Sud. De fait, l'Union européenne cherche désespérément davantage de solidarité entre ses membres ${ }^{3}$.

\footnotetext{
${ }^{3}$ Un plan de relance de 750 milliards d'euros (loin de faire l'unanimité) a par exemple été annoncé par la Commission européenne. Ce plan, dont les sommes seraient abondées au budget européen (qui ne représente jusqu'à présent qu'un peu plus de $1 \%$ du PIB européen) est sensé aller dans le sens d'une Europe plus intégrée, plus solidaire et plus fédéraliste. Dette commune, subventions (plutôt que prêts) et même la possibilité d'impôts communautaires sont évoqués. Ce plan serait financé par quelques mesures pourtant longtemps rejetées par les
} 
Les organisations économiques internationales nées de la dernière guerre mondiale ont perdu de leur influence et l'Organisation Mondiale du Commerce reste désarmée face aux exigences des États-Unis ou de la Chine. La finance internationale devient une boussole du bon fonctionnement des entreprises et un indicateur de la qualité des politiques économiques engagées par les États. Pourtant, l'histoire des crises économiques met en évidence que cette boussole est bien imprécise, incapable de mettre en évidence les bouleversements écologiques, sociétaux ou culturels d'aujourd'hui. Intéressée principalement par le profit immédiat et la spéculation, elle conduit à une fragmentation des chaînes de valeur (Moati et Mouhoud, 2005) et à une grande volatilité des flux financiers (Brunat, 2019) Aucune référence aux conséquences sociales et sociétales des opérations financières n'est alors engagée, puisque le système concurrentiel sans entrave est toujours supposé conduire à l'optimum. Le keynésianisme a progressivement perdu ses lettres de noblesse. L'État-nation n'est plus l'espace principal de la régulation, sauf dans les périodes de grande précarité sécuritaire et de crise économique. Si le capitalisme mondialisé a rendu l'action publique réactive, celle-ci n'est plus proactive. Il est vrai que Keynes a souvent contesté l'efficacité du capitalisme. "Le capitalisme international, aujourd'hui en décadence, aux mains duquel nous nous sommes trouvés après la guerre, n'est pas une réussite. Il est dénué d'intelligence, de beauté, de justice, de vertu, et il ne tient pas ses promesses. En bref, il nous déplaît et nous commençons à le mépriser. Mais quand nous nous demandons par quoi le remplacer, nous sommes extrêmement perplexes » (Keynes, 1932, p.203).

Après le rejet du système keynésien et de toute intervention de l'État (Lucas, 1977) et le triomphe du monétarisme de Milton Friedman, appliqué politiquement par l'Administration américaine de Ronald Reagan, la stabilité des prix, l'économie de l'offre et le libre jeu du marché se sont substitués à la doxa keynésienne, faisant fi par la même aux conclusions du Rapport Meadows du Club de Rome sur les limites de la croissance (Morin, 2020). Les politiques du "small state» dans une économie soumise au marché se sont imposées. Dès lors, elles ont rendu et rendent les États pingres, à la recherche de la réduction des coûts. Il est vrai que l'État a connu une augmentation importante de ses prélèvements obligatoires, en vue de réduire les aléas sociaux, médicaux de ses citoyens. Cela a été rendu nécessaire par l'augmentation des inégalités sociales depuis près d'un demi-siècle. Du fait des inégalités sociales croissantes et de la répugnance des ultra-riches à participer au financement par l'impôt des actions régaliennes et de redistribution des ressources, les États se sont endettés, notamment lorsqu'ils ont dû aussi intervenir pour financer le système bancaire et financier lors de la crise de 2008-2009.

Les États se sont fixé des normes statistiques de performance pour gouverner, ils se sont engagés dans une gestion de calcul et de modèles aux hypothèses souvent discutables. La croissance du PIB est devenue la norme d'excellence de la politique économique, sans s'interroger sur son contenu. Comme la monnaie, la croissance du PIB est devenue un équivalent général de la valeur de la gestion d'un État. Une politique des chiffres a été mise en place, concernant par exemple la norme d'un endettement public inférieur à $3 \%$ du PIB. Les résultats obtenus ne sont pas à la hauteur de l'objectif, mais ils permettent aux gouvernements de conduire une politique restrictive qui concerne d'abord le financement des services publics, au détriment des acteurs économiques qui en ont le plus besoin.

De manière plus précise, il convient de rappeler que le taux de croissance du PIB prend en compte principalement les opérations marchandes. Au moment de la création du PIB, Simon Kuznets (1930) et Colin Clark (1932) se sont interrogés sur le point de savoir s'il fallait calculer la mesure du bien-être (welfare) ou simplement l'activité économique ellemême. Le second agrégat a été choisi, car après la guerre et la crise de 1929, un plan de

États frugaux : une taxation augmentée et harmonisée des flux financiers, une taxe sur les déchets plastiques, les émissions de $\mathrm{CO} 2$ et les grandes firmes de type GAFAM... 
reconstruction économique était prioritaire. Il fallait alors exiger des agents économiques, notamment des ménages, d'engager des efforts particuliers pour sortir de la crise. La notion de bien-être pouvait attendre. Conscient de ses lacunes, son créateur l'avait présenté comme un indicateur de l'activité productive et avait mis en garde contre les risques d'utilisation abusive (Daly et Cobb, 1989). Le PIB est un indicateur particulier qui ne peut mesurer et encore moins représenter seul l'efficacité économique collective d'un pays. Il néglige les patrimoines, les coûts de l'environnement, le gaspillage des ressources naturelles, les inégalités sociales ou la violence de la production (Guilhaudis, Fontanel, 2019). Il peut être nourri de productions très éloignées des demandes sociales et des besoins réels des populations. Il relaie les informations trafiquées des firmes multinationales enclines à optimiser leurs profits en maquillant les chaînes de valeur réelles en vue de réduire leurs taxes. Enfin, la lutte compétitive vers le «moins disant» social auxquels sont soumis les États conduit à réduire les avantages collectifs des citoyens, au profit des bénéfices après impôts des firmes. Le PIB peut augmenter avec une réduction des libertés, la précarité et la violence des conditions de travail. Une augmentation des salaires des fonctionnaires est perçue comme un coût pour l'État, en omettant son antonyme l'amélioration des conditions de vie des salariés. En outre, les comparaisons internationales des chiffres concernant le PIB par habitant ou les personnes actives se font sans référence aux différences d'éthique, de civilisation, de coutumes, ou de qualité de vie. Les statistiques fournies en termes de parité de pouvoir d'achat ne manquent pas d'intérêt, mais elles sont aussi directement reliées au seul domaine marchand, lequel ne représente qu'une partie de l'activité individuelle et collective des hommes (Donsimoni, Fontanel, 2019).

J.M. Keynes annonçait il y a près d'un siècle qu'en 2030, les hommes pourraient travailler trois heures par jour et se consacrer à d'autres activités plus exaltantes (Keynes, 1932). Or, les désirs matériels se sont accrus. L'utilitarisme économique l'emporte sur le social. Selon Michel Foucault, le marché établit un ordre des valeurs qui donne lieu à une demande infinie, alors que le système de production ne peut être que fini (Foucault, 2001). La fabrique à frustration est alors mise en place, sous fond de cupidité organisée et même justifiée. Le conflit est latent. "Deux désirs qui convergent sur le même objet se font mutuellement obstacle. Toute mimesis portant sur le désir débouche automatiquement sur le conflit » (Girard, 2007, p 216). Et, dans ce contexte, l'État doit être à la fois cet arbitre et cet acteur qui permet de maintenir le lien entre les personnes physiques et morales, à l'intérieur et avec l'extérieur de son territoire.

Avec la pandémie et ses contraintes de production, il faut relancer la "machine » économique en panne, mais pour un pays sans ressources naturelles et avec une industrie très affaiblie, les règles du libre-échange ne permettront guère de lancer un nouveau «New Deal » keynésien sans volonté politique (Rifkin, 2019). La plupart des États ont perdu leurs anciennes compétences industrielles. Pour des raisons de sécurité nationale, les relocalisations de certaines activités économiques essentielles semblent pourtant nécessaires, afin d'éviter les ruptures d'approvisionnement. A la fin de la seconde guerre mondiale, des innovations sociales ont été mises en place en vue de réduire la précarité dans un monde de concurrence. Aujourd'hui, avec l'ouverture internationale, ces avantages sont contestés, ils sont perçus comme des coûts pour les entreprises, les fragilisant ainsi face à la concurrence internationale, ce qui conduit les entreprises à se délocaliser dans les pays socialement "moins-disants". Toute augmentation de salaire est alors perçue comme un coût plutôt que comme une avancée sociale au service du développement humain et du respect du niveau et de l'espérance vie des offreurs de travail.

Dans le système d'aujourd'hui, le travail d'un dirigeant d'entreprise consiste à faire gagner le plus d'argent possible à ses actionnaires, quels qu'en soient les coûts sociaux. Il faut alors chercher à réinventer l'entreprise et le management. "Les entreprises, les investisseurs 
et les écoles de management, tous sont incités à repenser leur approche de la création de la valeur, ainsi que leur contribution à la société : leur fameuse "raison d'être ". Tous sont incités à réinventer leurs systèmes et leurs pratiques de management en intégrant le principe de réciprocité vis-à-vis de l'écosystème dans lequel ils opèrent... Une économie de la réciprocité plus humaine et respectueuse de l'environnement peut être plus rentable qu'une économie financière " (Roche, 2019, page 11) Le capitalisme peut, en effet, s'effondrer avec la montée des inégalités, les tensions sociales et les questions environnementales. Il faut repenser l'idée même de la création de valeur en la rapprochant de la "raison d'être ». Le monde n'est pas qu'un univers de jeu, de gagnants et de perdants, ce système a tendance à détruire progressivement les fondements de toute vie sur terre, avec le réchauffement climatique menaçant.

Négligeant la réalité du principe de «no bridge» entre micro et macroéconomie, les règles du management des entreprises ont été appliquées à l'État dans le cadre de la rationalisation néolibérale. Paul Krugman (1996) a pourtant rappelé qu'un pays n'est pas une entreprise. La "reprise" du vocabulaire managérial pour évaluer les performances d'une nation est une des obsessions dangereuses de notre époque. La croissance est devenue la religion économique du monde moderne. Même le «Business roundtable», organisation du lobbying des grandes entreprises, demande aujourd'hui une redéfinition de l'objet de l'entreprise, en vue de sortir du dogme de la maximisation du profit financier. Il faut repenser les ressorts de la croissance. Le capital financier est surabondant et prédateur, l'entreprise comme entité de production en souffre au profit des actionnaires et des dirigeants. Un management moderne doit tenir compte de tout l'apport de l'entreprise dans la société, sans se référer uniquement aux valeurs financières. La conquête violente du profit maximisé devient dangereuse pour la démocratie, l'économie de marché et la justice productive. Sans un Etat fort, assurant le bon fonctionnement de l'économie nationale, le terreau du profit risque de se tarir. Les grandes entreprises multinationales jouent à court terme avec les Etats pour obtenir les plus grands avantages, à la fois pour assurer la pérennité de leur entreprise, mais aussi pour accroître leurs rémunérations. La question est alors de savoir si l'Etat peut assurer ses fonctions régaliennes face à des firmes qui cherchent à éviter de les financer.

\section{2) La puissance des entreprises et l'affaiblissement des valeurs régaliennes}

Les économies nationales ne peuvent pas fonctionner sans un État capable de faire respecter un cadre légal clair et stable. Le progrès économique n'est pas issu des seuls mécanismes du marché, l'intervention de l'État est nécessaire. Trois arguments concrets viennent étayer cette assertion :

- L'État assure et rassure, notamment contre le terrorisme ou les convoitises extérieures. En effet, la défense nationale d'un pays ne se limite pas au seul domaine militaire. La dépendance nationale à la fourniture de biens et services suppose des accords interétatiques, des projets collectifs contrôlés et des négociations internationales que le marché n'est pas à même d'assumer. Les conflits de toute nature opposant deux pays peuvent conduire à une "guerre économique » orchestrée par les pouvoirs publics, laquelle affaiblit les pays belligérants. Ainsi, la dépendance des pays occidentaux aux services des GAFAM ne manque pas d'inquiéter si l'on considère qu'ils sont clairement utilisés à la fois par les forces armées et les entreprises localisées sur le territoire national. La liberté commerciale et industrielle conduit alors clairement à une dépendance aux décisions d'entreprises et d'un État puissants et dominants (Fontanel et Sushcheva, 2019).

- Ensuite, les forces du marché revendiquent le respect du droit de propriété défini par le droit romain, avec ses trois caractéristiques, «l'usus, le fructus et l'abusus ». Or, l'abus de la propriété individuelle pose des problèmes spécifiques à la collectivité que celle-ci ne peut 
plus accepter (pollution, changement de climat, situation de monopole, exploitation du travail, etc.). Dans ce contexte l'État a le devoir de protéger les citoyens. C'est dans le cadre de ses fonctions régaliennes qu'il doit proposer des biens publics comme la justice, la police, l'armée ou encore l'éducation et la santé. Pour accomplir cette fonction, les pouvoirs publics disposent de ressources, notamment par les impôts. Or, les économistes d'inspiration libérale considèrent souvent que les impôts payés par les entreprises lèsent la croissance nationale. Pourtant, les revenus des entreprises sont d'abord le résultat de lois qui les protègent et leur permettent de prospérer.

- Enfin, la liberté sans morale constitue un facteur de crise économique. L'essence de la crise de 2008 se trouve dans les opérations des acteurs bancaires et financiers sur le marché de l'immobilier aux USA. La spéculation a été jugée responsable, mais comme elle n'a pas été punie, elle n'est donc pas coupable. Or, de nombreux agents bancaires et financiers ont parié cyniquement sur l'effondrement du système pour accroître leurs fortunes. Dans ce contexte, l'aléa moral joue pleinement sa fonction désorganisatrice. Les spéculateurs n'ont aucun respect pour les normes éthiques et même parfois pour le droit. Les banques ont alors quasiment vendu des produits de recel, avec les produits dérivés. La Federal Reserve System (FED), banque centrale des États-Unis, n'a pas souhaité intervenir face à ces montages financiers peu scrupuleux et toutes les barrières de protections antérieures ont été ainsi démantelées. La stabilité intrinsèque du système d'économie de marché n'est pas vraiment établie (Minsky, 2008). Lorsqu'un indicateur s'écarte de ses valeurs antérieures, le risque de crise est accru par la peur du risque de ceux qui ont des intérêts fondés sur la spéculation. Les paris spéculatifs transforment les positions couvertes en des positions aléatoires, mais très profitables. Dans ce cas, la stabilité positive du spéculateur qui ne veut pas perdre d'argent, alimente l'instabilité des structures financières et donc du système capitaliste. Ce système de prévarication généralisée n'a guère permis de définir des responsabilités claires, au regard des complicités objectives entre les opérateurs publics et privés. Il est alors possible de constater que les Etats sont très dépendants des banques, d'autant plus qu'ils sont endettés. La peur de la crise économique est d'autant plus forte que les Etats ont répondu positivement à la déréglementation, à la désintermédiation et au décloisonnement des marchés nationaux et internationaux ${ }^{4}$.

Dans ce contexte, les banques ont aussi accompagné les spéculateurs à échapper aux systèmes fiscaux nationaux, accroissant ainsi les difficultés des pouvoirs publics à percevoir les ressources attendues de l'impôt. Dans cet espace virtuel de libre échange, l'optimisation fiscale permet d'accroître les bénéfices des très grandes firmes, et l'évasion fiscale menace aujourd'hui la stabilité même des ressources des États. Or, paradoxalement, le manque de ressources financières est un des leitmotivs de tous les discours des responsables politiques. Et dans un tel contexte, les financements de l'enseignement, de la recherche, de la santé du secteur public et de la protection sociale sont affaiblis par les compressions de crédits qui empêchent la réalisation des objectifs collectifs. L'État est dépouillé d'une partie de ses revenus légitimes et pourtant, l'idée dominante reste que les impôts sont déjà trop élevés et réduisent la croissance. Le discours colle dans la pensée des citoyens comme un slogan, éloigné des prétentions scientifiques qu'il relaie. De fait, le travail d'un dirigeant d'une grande firme consiste à faire gagner le plus d'argent à ses actionnaires, sans se préoccuper de

\footnotetext{
${ }^{4}$ L'influence des préceptes libéraux du Consensus de Washington dès le début des années 90 -déréglementation, stabilisation macroéconomique, privatisation notamment- appliqués en premier lieu dans les économies en difficulté du fait de leur endettement comme en Amérique du sud, puis en Russie en rupture brutale avec le système de type soviétique (Brunat et Fontanel, 2015), a été considérable. Une majorité d'États et d'Organisations Internationale, comme la Banque Mondiale et le Fonds Monétaire International ont été profondément influencés par la pensée néoclassique, dominante et majoritaire, pour orchestrer ce que d'aucuns ont défini comme la fin de l'histoire (Fukuyama, 1992).
} 
l'impact de son action sur l'ensemble de la communauté nationale ou internationale. Il n'est jugé que sur la capacité à développer l'entreprise, quels que soient les coûts sociaux supportables, et de fournir des revenus conséquents sous forme de dividendes ou d'autofinancement aux actionnaires.

Les pouvoirs publics justifient cette situation par la théorie du «ruissellement» (trickle-down theory), qui considère que les revenus des plus riches contribuent à la croissance économique nationale et à la création d'emplois. Pour David Stockman, Directeur du budget sous la Présidence de Ronald Reagan, il est nécessaire d'accorder des réductions d'impôts aux tranches supérieures, aux individus les plus riches et aux plus grandes entreprises, et laisser ainsi les bons effets « ruisseler» à travers l'économie au bénéfice de tout le monde' Il ajoute ensuite qu'il est difficile de «vendre » l'idée du ruissellement. Dans ces conditions, la théorie de l'offre était la seule formule possible pour justifier et mener une véritable politique fiscale du ruissellement, laquelle était l'objectif réel (Greider, 1981). Dans ce contexte, la richesse produite par les plus riches profite, avec un décalage de temps plus ou moins important, aux plus pauvres. Cette «fable» (Parienty, 2018) a été présentée depuis près d'un demi siècle, mais les statistiques économiques sur les revenus des personnes montrent un écart croissant entre les deux extrémités des revenus, comme ont pu le démontrer Saez et Zucman (2020). Une croissance nationale forte peut être à la fois inéquitable et inégalitaire. La théorie du "ruissellement» n'a pas réellement été défendue par les théories économiques elles-mêmes, mais elle a souvent été présentée et défendue par les couches riches de la population et par certains gouvernements (Reagan, Thatcher), afin d'inciter les acteurs économiques des classes moyennes et pauvres d'accepter leur situation tout en témoignant de la reconnaissance pour ceux qui, en s'enrichissant, les laisse espérer d'éventuelles miettes du gâteau dans un futur indéfini.

L'horizon temps des décideurs économiques est très hétérogène. L'économie marchande s'inscrit dans un espace de court terme, au regard des évolutions rapides qui secouent le champ de leurs compétences, de leurs contraintes de profit et de leurs parts de marchés. Elle ne donne que des informations parcellaires, partiales, qui permettent de faire d'importants profits pour une entreprise, mais parfois au détriment de la santé et du confort des populations. Au contraire, les pouvoirs publics doivent lutter contre les incertitudes du long terme. Le problème du réchauffement du climat induit par les émissions de gaz à effet de serre ne peut pas être traité par l'économie marchande. L'État a pour vocation de prendre en charge ces menaces. Or, les entreprises polluantes ne souhaitent guère réduire leurs profits et elles agissent en lobbyistes pour contrecarrer les actions de l'État sous peine d'une augmentation du chômage ou de nouvelles difficultés économiques laissant libre champ à la concurrence étrangère.

\section{3) Des théories économiques et des politiques en forme de slogans}

Plusieurs slogans en forme de vérités scientifiques sont supposés indiscutables dans la société. D'abord, l'économie est une science, tout comme le management. Puis, l'économie de marché ne peut se développer sans démocratie. Le marché fonctionne avec fluidité, sans le poids économique excessif d'États puissants. Enfin, en cas d'un effet exogène, la solidarité politique citoyenne doit intervenir.

\section{discutables}

\section{1) Les hypothèses dominantes de la «science économique» sont}

En simplifiant, l'économie dominante considère que le marché produit un équilibre économique sans frictions du monde globalisé, sur la base d'hypothèses aux conséquences 
rarement élucidées lorsqu'elles s'éloignent de l'observation. Il est certes toujours possible d'amender progressivement le modèle et certaines hypothèses, mais il faut toujours partir du modèle de base ; si ce n'est pas le cas, il est reproché à l'analyse son manque de rigueur. Il faut accepter de s'inscrire dans le schéma préétabli par l'une ou l'autre des écoles. L'économie n'échappe pas à l'idéologie et à une certaine conception de l'homme et de son environnement social (Precht, 2010). C'est pourquoi on peut considérer qu'elle est aussi fondamentalement politique, même si des outils scientifiques doivent être rendus disponibles pour proposer ou vérifier l'efficacité des actions individuelles et sociales entreprises. C'est ainsi que, face à une crise économique, les économistes libéraux ne remettent pas en cause le système lui-même, mais l'inadaptation des politiques et des réglementations publiques. Par exemple, la crise financière de 2008 avait été annoncée par plusieurs économistes hétérodoxes, mais leurs analyses étaient alors présentées comme de simples critiques politiques ou idéologiques. "Il y avait des exceptions à ces tendances : quelques économistes contestaient le postulat du comportement rationnel, doutaient fort qu'on pût faire confiance aux marchés financiers et rappelaient la longue histoire des crises financières qui avaient eu des conséquences économiques dévastatrices. Mais ils nageaient à contre-courant, incapables d'avancer face à l'autosatisfaction omniprésente, et avec le recul, absurde » (Krugman, 2009). Dans l'esprit des analystes les plus traditionnels, ce n'est pas la modélisation qu'il faut contester, c'est l'incapacité du monde réel à se conformer aux hypothèses du système formalisé ainsi construit.

Le régime d'accumulation postfordiste prend ses racines au début des années 1980, notamment à la suite des mesures de libéralisation et de déréglementation financière. François Chesnais (2009) parle de "régime d'accumulation financiarisé mondial" avec des taux de croissance assez bas observés dans de nombreuses régions du monde "au centre et à la périphérie", la montée d'un chômage de masse, l'accentuation des inégalités entre pays, et des inégalités de revenus et de conditions d'existence à l'intérieur de chacun d'eux pris individuellement. La situation est telle que même si la Banque Mondiale observe un recul de l'extrême pauvreté dans la période récente ${ }^{5}$, les situations d'inégalités se sont aggravées. En particulier, ce régime d'accumulation conduit à un recul de la civilisation parce que l'humain n'est pas suffisamment valorisé par rapport à une efficacité économique qui ne profite pas à l'ensemble de la population. Par ailleurs, il ne met pas suffisamment en évidence la stagnation des revenus des classes moyennes et pauvres, dans un contexte de croissance qui ne bénéficie in fine, depuis 40 ans, qu'aux seules classes riches et ultra-riches (Saez et Zucman, 2020). L'extrême pauvreté a été partiellement réduite par le seul déclassement progressif d'une frange importante des classes moyennes et pauvres.

Or les modèles économiques omettent les rapports de force entre les États, les conflits politiques ou armés, l'existence des paradis fiscaux (Fontanel, 2016) le jeu silencieux et obscur des firmes multinationales dans la gestion et la redistribution de leurs profits, l'émergence de nouvelles règles communautaires ou internationales qui ont pourtant pour vocation de modifier la « donne » concernant l'évolution du système mondial globalisé. Tout modèle a pour objectif de construire une représentation simplifiée de la réalité. Cependant, lorsqu'un système formalisé est mis en place et produit des résultats, il est nécessaire de s'interroger sur l'influence de ses hypothèses et des facteurs délibérément omis sur les résultats du modèle. Or, cette seconde étape est trop souvent négligée, car les hypothèses sont devenues des normes «scientifiques", qui se suffisent à elles-mêmes et donc qu'il est

\footnotetext{
5 "Ces dernières décennies ont été marquées par des progrès importants dans la réduction de la pauvreté, qui constitue aujourd'hui le premier des Objectifs de développement durable fixés par les Nations Unies. D'après les estimations les plus récentes, $10 \%$ de la population mondiale vivait avec moins de 1,90 dollar par jour en 2015, ce qui représentait 734 millions d'habitants. Ce taux atteignait près de $36 \%$ en 1990, soit 1,9 milliard de personnes", https:/www.banquemondiale.org/fr/topic/poverty/overview.
} 
difficile de remettre en cause ${ }^{6}$. Le modèle devient un «jeu» intellectuel à partir duquel se formeront les avenirs professionnels des épigones les plus brillants. Pour James Galbraith, "l'objectif essentiel de ces formules mathématiques n'est pas d'instruire, ni de plaire, mais d'intimider » (Galbraith, 2014, page 79)

\section{2) Un concept racorni de démocratie}

Le capitaliste d'aujourd'hui cumule le profit et le pouvoir. Avec la fumeuse théorie du ruissellement, il défend l'idée selon laquelle l'intérêt général se confond avec son propre intérêt. Il n'est pas cupide, il cherche la maximation personnelle d'un profit qui profitera à terme à l'ensemble de la société. Thomas Piketty a montré que la tendance des fortunes à se concentrer est inhérente au système capitaliste, sauf pendant les périodes de guerre. Les valeurs démocratiques sont affaiblies par les puissances de l'argent qui peuvent agir secrètement sur la politique de l'État (Piketty, 2013). Les firmes multinationales exercent une influence considérable sur les institutions publiques, car elles ont le pouvoir de financer les campagnes électorales, de contrôler l'information (télévision, journaux, internet, réseaux sociaux, données des États) et d'exercer un lobbying efficace dans toutes les instances nationales et internationales. Elles peuvent s'offrir les meilleures intelligences pour défendre leurs intérêts, grâce aux passages d'un emploi du service public vers le secteur privé, et vice versa, de leurs affidés.

Le caractère difficilement justifiable des différences de revenus et de patrimoines constitue une menace grave contre la démocratie. Aux États-Unis, les plus riches paient proportionnellement de moins en moins d'impôts par rapport à la classe moyenne. Les salaires des classes populaires ont stagné depuis des années, leur endettement s'est accru ainsi que la précarité de leurs emplois. Le triomphe de l'injustice fiscale est un déni de justice, de collaboration internationale et de démocratie. Sans impôt, il n'y a pas de destinée commune. Dans ces conditions, la démocratie est en crise très profonde, et une ploutocratie semble s'installer partout dans le monde. Le déterminisme du milieu et "l'Habitus" défendus par Pierre Bourdieu pour décrire la distribution du pouvoir (Bourdieu, 1989), n'ont pas été sérieusement corrigés par les systèmes publics d'éducation au sein des pays avancés. Cependant, là où les services publics ont déserté, naissent les "gilets jaunes". Le triomphe de l'injustice est aujourd'hui patent. Les petites entreprises sont dominées par les grandes firmes multinationales qui bénéficient d'avantages fiscaux dont elles ne peuvent vraiment pas bénéficier. Le système de crises économiques conduit à l'essor d'une violence de fond qui peut remettre en cause toutes les valeurs collectives, tout le lien social qui fondent une Nation et un système économique. On assiste à un processus de déliaison, de délitement du lien social, véritable dérèglement de la logique démocratique.

\section{3) L’aléa dans la science économique}

La théorie des «cygnes noirs» met en avant l'existence inopinée de certains phénomènes qui ne sont pas prévisibles dans le temps et dans l'espace même si l'on sait qu'ils peuvent quand même se produire, sans en comprendre le déclencheur (Taleb, 2010). C'est le cas des crises financières ${ }^{7}$, fondées sur les comportements spéculatifs. De même, la théorie

\footnotetext{
${ }^{6}$ La Terre n'est pas plate, comme le pensaient en leur temps les scientifiques, les religieux ou les philosophes face à Galilée !

${ }^{7}$ Edgar Morin a dédié de nombreux travaux à "la complexité" en s'intéressant aux incertitudes de l'homme sur sa propre nature, en cherchant à mettre en évidence les complexités de la physique, de la pensée biologique, et de l'épistémologie. Il se concentre non pas sur l'ordre mais sur le désordre des choses. Il convient aussi de rappeler René Thom (1983) qui est l'un des pères de la 'théorie des catastrophes'. Il montre que des contextes particuliers
} 
des « longues traînes » considère que la distribution des erreurs répond aux règles de la loi normale, mais il est difficile de déterminer leur fréquence et surtout le moment où les instabilités du système produisent la crise $^{8}$. Les risques sont, a priori, difficilement calculables, notamment la profondeur de la crise, ses localisations, sa durée. Au moment de la crise, l'État est jugé responsable et tous les acteurs économiques lui demandent alors de trouver les solutions qu'il paiera plus tard d'un endettement croissant qui lui sera évidemment reproché comme l'expression d'une mauvaise gestion des biens publics nationaux.

\section{4) L’importance des rapports de force des États}

En 1995, avec la création de l'OMC, le commerce «pacifique » en toute liberté est devenu la base de la vie sociétale mondiale (Fontanel, 1995). Les firmes multinationales, libérées des exigences de chaque État, pouvaient alors imposer leurs lois commerciales positives et augmenter le PIB mondial au profit de l'écoumène. Washington était devenu le principal gendarme du monde, avec des dépenses militaires (Otan compris) à sa main près de deux fois supérieures à celles du reste du monde. L'histoire était belle, un conte de fées. Cependant, dans le cadre de l'affaire nucléaire iranienne, la Maison Blanche a imposé sa loi unique, avec violence, malgré les recours devant une OMC qui n'a pas osé condamner le pays qui a été son parrain sous les fonds baptismaux du libre-échange. Toujours est-il que l'OMC est aujourd'hui paralysée par une administration américaine qui ne supporte pas la moindre résistance à ses intérêts commerciaux. Désormais l'heure est au divorce et au désengagement des Etats-Unis de l'OMC, officialisés (mais pour combien de temps ou à quelle échéance) par le Président Trump en juillet 2020. Avec la politique du "benign neglect" ${ }^{\prime \prime}$ toujours d'actualité, les droits de douane établis en «caroussels », l'application des lois américaines extraterritoriales, les forces militaires ou le contrôle quasi mondial de l'information par les GAFAM (Fontanel et Sushcheva, 2019), Washington impose sa puissance aux entreprises ou aux États. La Chine déploie un capitalisme aux ordres politiques de l'État central, sans respect pour les droits de l'homme, dans le cadre d'une économie nationale contrôlée de l'intérieur par les membres du Parti au pouvoir depuis 1949. La faiblesse de l'Europe naît de ses divisions, de son histoire, d'objectifs politiques et commerciaux différents, d'idéologies nationalistes et de conceptions nationales encore étroites. La guerre économique est clandestine, mais elle s'exprime pour maintenir les positions dominantes des États et des firmes.

Aujourd'hui, les États des «paradis fiscaux » commercialisent leur souveraineté, en offrant aux firmes multinationales des avantages fiscaux importants. Ce sont d'ailleurs les mêmes États qui, au nom de la rigueur financière, ne souhaitent pas soutenir les pays qu'ils ont parfois ainsi délestés de leurs plus gros contribuables. Lorsqu'il y a crise, les États interviennent et injectent des financements dans les banques pour relancer l'économie. C'est

et complexes peuvent déboucher sur des discontinuités brutales (chaos) et des enchainements difficilement prévisibles.

${ }^{8}$ Voir l'entretien du père de la théorie des "longues traines" appliquée notamment aux questions financières, The Father of long tails, interview avec Benoît Mandelbrot (2010) sur le site de Edge (www.edge.org), https://www.edge.org/3rd_culture/obrist10/obrist10_index.html

9 Le "benign neglect" est un "laisser-aller serein" quant à la politique de change du dollar - monnaie internationale depuis les Accords de Bretton Woods de 1944 et la mise en place du système de change-or. La formule s'impose avec l'émergence du régime des changes flottants dans les années 70, depuis que l'Administration du président Richard Nixon a décidé de suspendre la valeur fixe du dollar en or. Les Etats-Unis laissent depuis fluctuer le dollar sans interventions publiques fortes. Attribuée parfois à John Connally ancien Secrétaire d'Etat au Trésor, parfois à Paul Volker, ancien Gouverneur de la FED, les célèbres mots "le dollar est notre monnaie, mais c'est votre problème" signifie que les Etats-Unis considèrent que le dollar est bien la seule véritable monnaie internationale de réserves et de transactions et qu'il appartient de facto au reste du monde de se soucier des parités. 
une période d'austérité pour les citoyens, mais les grandes entreprises réussissent alors à augmenter de nouveau leurs profits et les dividendes de leurs actionnaires. Le système n'échappe pas à la critique de prévarication et d'un sentiment collectif et individuel de nonresponsabilité devant les catastrophes qui se préparent.

\section{Conclusion}

La maximisation du taux de profit, l'hypothèse de la rationalité parfaite des agents économiques et la financiarisation du capitalisme débouchent sur la fragmentation des chaînes de valeur, l'optimisation et l'évasion fiscale et le creusement des inégalités. Par la recherche sans fin et sans limite "d'une maximisation du profit" et de la primauté de l'intérêt individuel, le modèle d'accumulation dominant néglige les fractures sociales et la mutilation des conditions de vie environnementales du monde non marchand. L'approche cryptomathématique et financière, considérée comme l'instrument dominant de la pensée économique même, justifie toutes les actions qui conduisent à la croissance économique du PIB par la maximation du profit. L'économie politique a laissé le champ libre à une science économique humainement désincarnée, sans lien avec la diversité des civilisations ou des croyances religieuses ou philosophiques, qui justifie les contraintes du travail, les inégalités sociales ou le retour de la précarité comme fondement d'une nouvelle " armée de réserve ». Un développement économique humaniste, tenant compte de la vie des personnes à court et à très long terme, doit être envisagé, face aux difficultés économiques et sociales de nombre d'individus, au réchauffement climatique, à l'épuisement progressif des ressources naturelles ou au non respect des droits de l'homme et de la femme dans de nombreux États. La pandémie de la Covid-19 met en évidence l'importance des liens entre les hommes, leur fragilité et la nécessité de construire des liens sociaux plus solidaires et conviviaux. Il s'agit de replacer l'être humain au cœur des priorités de la gouvernance aux niveaux local, national et mondial. Dans cette lignée, la transition écologique est devenue incontournable. En outre, l'exacerbation de la fragmentation sociale résultant de la pandémie de la Covid-19, met encore plus l'accent sur l'impératif d'équité sociale pour faire avancer l'écologie politique (Aglietta, 2019), accepter le principe d'une transformation des comportements économiques, donner de la valeur aux solidarités et réduire l'addiction à la possession indéfinie des biens et services marchands.

\section{Bibliographie}

Aglietta, M. (sous la direction) (2019), Capitalisme : le temps des ruptures, Odile Jacob, Paris

Bourdieu, P. (1989), La noblesse d'État, Minuit, Paris

Brunat, E. (2019), Basculement du monde, développement humain et paix, in «Pour un art de la paix », École de la Paix, Grenoble

Brunat, E. (2020), Les entreprises doivent intégrer la géopolitique, Eco Savoie Mont Blanc, hors série, mars

Brunat E., Fontanel J. (2015), L'économie de la Russie, les grands défis à relever, AFRI, Annuaire Français de Relations Internationales, Paris

Case, A., Deaton, A. (2020), Deaths of Despair and the future of capitalism, Princeton University Press, Princeton

Chesnais, F. (2009), Mondialisation du capital \& régime d'accumulation à dominante financière 29 novembre, charro1010.worldpress.com

Clark C. (1932), The National Income, 1924-31, Macmillan \& Co. 
Coyle, D. (2014), Is GDP still useful? Better Life Index, May 16

http://www.oecdbetterlifeindex.org/blog/is-gdp-still-useful.htm

Daly, H., Cobb, J. (Jr) (1989), For the Common Good: Redirecting the Economy toward Community, the Environment and a Sustainable Future, Beacon Press, Boston.

Donsimoni, M., Fontanel, J. (2019), Les ambiguïtés de la mesure de la puissance économique des États par les parités de pouvoir d'achat, AFRI, Annuaire Français des Relations Internationale, Paris

Fontanel, J. (1995), Organisations économiques internationales, Masson, 1995.

Fontanel, J. (2016), Paradis fiscaux, pays filous. La fuite organisée des impôts vers les pays complices. L'Harmattan, Paris

Fontanel, J. (2020), La troisième révolution industrielle. Une question de survie? PSEI, revel.unice.fr

Fontanel, J., Sushcheva, N. (2019), La puissance des GAFAM. AFRI, Annuaire Français des Relations Internationales, Paris

Foucault, M. (2001), Dits et écrits, Gallimard, Paris

Fukuyama, F. (1992), The end of History and the last man, Macmillan, New York

Galbraith, J. (2014), La grande Crise, Le Seuil, Paris

Girard, R. (2007), La violence et le sacré, Grasset, Paris.

Greider, W. (1981), The education of David Stockman, Interview in The Atlantic, December.

Guilhaudis, J-F., Fontanel, J. (2019), Les effets "pervers" de l'usage du PIB pour la décision politique et les relations internationales, AFRI, Annuaire Français des Relations Internationales, Paris

Keynes, J.M. (1932), La pauvreté dans l'abondance, Gallimard, 2007, Paris

Krugman, P. (1996), A country is not a company, Harvard Business Review, Jan.-Feb.

Krugman, P. (2009), How Did the Economists Get it So Wrong? », New York Times, Sunday Magazine, September

Kuznets, S. (1930), Secular Movements in Production and Prices, Houghton-Mifflin, Boston and New York

Lucas, R.E. (1977), Understanding business Cycles, Carnegie-Rochester, Conference Series on Public Policy, Vol. 5

Minsky, H.P. (2008), Stabilizing an Unstable Economy, McGraw-Hill, NewYork

Moati, P., Mouhoud, E.M. (2005), Les nouvelles logiques de décomposition internationale des processus productifs, Revue d'Economie Politique, ${ }^{\circ} 5$, vol. 115

Morin, E. (2020), Sur la crise, Flammarion, " Champs Essais », Paris

Parienty, A. (2018), Le mythe de la " théorie du ruissellement », La Découverte, Paris

Piketty, T. (2013), Le Capital au $21^{e}$ siècle, Le Seuil, Paris

Piketty, T. (2019), Capital et idéologie, Le Seuil, Paris

Precht, R.D. (2010) Qui suis-je ? Et si je suis, combien?, Voyage en philosophie. Belfond. Paris.

Rifkin, J. (2019), Le New Deal vert mondial, Les liens qui libèrent, Paris

Roche, B. (2019) Réinventer l'entreprise et le management, Le Monde, 12 décembre

Saez, E., Zucman, G. (2020), Le triomphe de l'injustice. Richesse, évasion fiscale et démocratie, Le Seuil, Paris

Smith, A. (1776), La richesse des nations, Guillaumin, réédition de 1843 (première édition en 1776), Paris.

Smith, R., Fontanel, J. (2008), International security, defence economics and the powers of Nation. War, peace and security, 6, p.37à 52 .

Stiglitz, J, Sen, A., Fitoussi, J-P. (2009), The measurement of economic performance and social progress revisited, OFCE, Paris 
Taleb, N.N. (2010), The Black Swan: the impact of the highly improbable, 2nd Ed. (1st Ed. 2007), Penguin, Londres

Thom, R. (1983), Paraboles et catastrophes, Flammarion, Paris 